\title{
On Existence of Solitons for the 3rd Harmonic of a Light Beam in Planar Waveguides
}

\author{
V. CaO Long ${ }^{a}$, P.P. Goldstein ${ }^{b, *}$ And M. TrippenbaCh ${ }^{c}$ \\ ${ }^{a}$ Institute of Physics, University of Zielona Góra \\ Podgórna 50, 65-246 Zielona Góra, Poland \\ ${ }^{b}$ The Andrzej Soltan Institute for Nuclear Studies \\ Hoża 69, 00-681 Warsaw, Poland \\ ${ }^{c}$ Institute of Experimental Physics, Optics Division, Warsaw University \\ Hoża 69, 00-681 Warsaw, Poland
}

(Received December 4, 2009; in final form April 26, 2004)

\begin{abstract}
We consider equations governing propagation of a monochromatic laser beam coupled to its third harmonic in a nonlinear medium. The system proves to be non-integrable in the sense of Painlevé. However it is partially integrable for all values of its parameters. We further check the possibility of solving the equations by the Hirota bilinear method. The system is found to be solvable this way provided that the complex phase of the third harmonics is equal to tripled phase of the fundamental mode (modulo $\pi$ ) and also the amplitudes of these modes are in special proportion. This result corresponds to the previously known condition of existence of the sech soliton solutions. Furthermore, the Hirota scheme is found to work only for exact resonance, i.e. for the ratio of the dispersion coefficients equal to the ratio of frequencies. Finally, all these conditions may only be satisfied for single envelope solitons of the cubic Schrödinger type.
\end{abstract}

PACS numbers: 42.81.Dp

\section{Introduction}

Generation of harmonics is an inevitable companion to laser beam propagation through nonlinear media. Sometimes advantageous, it may also cause trouble,

*corresponding author; e-mail: Piotr.Goldstein@fuw.edu.pl 
by disturbing the fundamental mode. It is of crucial importance whether soliton pulses may persist in the conditions when the fundamental mode coexists with its higher harmonics. The present paper deals with such conditions for a model describing propagation of the third harmonic $[1,2]$ (some results on the second harmonic were presented in [3], a more comprehensive paper is in preparation). Our model will be a nonlinear optical medium with a cubic response. We start with the wave equation for the propagating electric field:

$$
\left(\nabla_{\perp}^{2}+\frac{\partial^{2}}{\partial z^{2}}-\frac{1}{c^{2}} \frac{\partial^{2}}{\partial t^{2}}\right) \boldsymbol{E}(\boldsymbol{x}, t)=\frac{4 \pi}{c^{2}} \frac{\partial^{2}}{\partial t^{2}}\left(\boldsymbol{P}_{\mathrm{L}}(x, t)+\boldsymbol{P}_{\mathrm{NL}}(\boldsymbol{x}, t)\right),
$$

where $\boldsymbol{P}_{\mathrm{L}}$ and $\boldsymbol{P}_{\mathrm{NL}}$ are linear and nonlinear polarization vectors, respectively. We are interested in the resonant interaction between a linearly polarized beam of frequency $\omega$ and its third harmonic, which is assumed to be identically polarized. We also assume that the beams propagate in a slab waveguide, then they have only one transverse dimension with the transverse laplacian $\nabla_{\perp}^{2}=\partial^{2} / \partial x^{2}$. As a result we obtain the scalar wave equation

$$
\left(\frac{\partial^{2}}{\partial x^{2}}+\frac{\partial^{2}}{\partial z^{2}}\right) E(x, z, t)-\frac{n^{2}}{c^{2}} \frac{\partial^{2}}{\partial t^{2}} E(x, z, t)=\frac{4 \pi}{c^{2}} \frac{\partial^{2}}{\partial t^{2}} P_{\mathrm{NL}}(x, z, t),
$$

where $n$ is the linear refractive index, and $P_{\mathrm{NL}}$ is the (third-order) nonlinear polarization. We consider for the fundamental and third harmonic

$$
E=\frac{1}{2}\left(E_{1} \exp \left[\mathrm{i}\left(k_{1} z-\omega t\right)\right]+E_{3} \exp \left[\mathrm{i}\left(k_{3} z-3 \omega t\right)\right]+\text { c.c. }\right)
$$

where $k_{j}=j \omega n_{j} / c, n_{j}=n(j \omega), j=1,3$. Substituting this electric field to the nonlinear polarization $P_{\mathrm{NL}}=\chi^{(3)} E^{3}$, where $\chi^{(3)}$ is a diagonal element of the third-order susceptibility, we obtain the following terms (taking the terms containing $\exp \left[\mathrm{i}\left(k_{1} z-\omega t\right)\right]$ and $\exp \left[\mathrm{i}\left(k_{3} z-3 \omega t\right)\right]$, correspondingly):

$$
\begin{aligned}
& P_{\mathrm{NL}}(\omega)=\frac{\chi^{(3)}}{8}\left[3\left|E_{1}\right|^{2} E_{1}+6\left|E_{3}\right|^{2} E_{1}+9 E_{1}^{* 2} E_{3} \exp (-\mathrm{i} \delta k z)\right] \exp \left[\mathrm{i}\left(k_{1} z-\omega t\right)\right], \\
& P_{\mathrm{NL}}(3 \omega)=\frac{\chi^{(3)}}{8}\left[3\left|E_{3}\right|^{2} E_{3}+6\left|E_{1}\right|^{2} E_{3}+E_{1}^{3} \exp (\mathrm{i} \delta k z)\right] \exp \left[\mathrm{i}\left(k_{3} z-3 \omega t\right)\right],(4)
\end{aligned}
$$

where $\delta k=3 k_{1}-k_{3}$. Substituting Eq. (4) into Eq. (1) and assuming that the envelope functions $E_{1}$ and $E_{3}$ are slowly varying, we find that all the temporal derivatives can be omitted. Furthermore we also assume

$$
\left|\frac{\partial^{2} E_{1}}{\partial z^{2}}\right| \ll\left|k_{1} \frac{\partial E_{1}}{\partial z}\right|
$$

and a similar condition for $E_{3}$. This leads us to a pair of coupled nonlinear equations. Introducing a beam width $x_{\mathrm{b}}$ and diffraction length $z_{\mathrm{d}}=2 x_{\mathrm{b}}^{2} k_{1}$ and therefore defining the dimensionless variables $z=z_{\mathrm{d}} Z$ and $x=x_{\mathrm{b}} X$ we obtain the following dimensionless equations: 


$$
\begin{aligned}
& \mathrm{i} \frac{\partial U}{\partial Z}+\frac{\partial^{2} U}{\partial X^{2}} \pm\left[\left(\frac{1}{9}|U|^{2}+2|W|^{2}\right) U+\frac{1}{4} U^{* 2} W\right]=0 \\
& \mathrm{i} \sigma \frac{\partial W}{\partial Z}+\frac{\partial^{2} W}{\partial X^{2}}-\Delta \sigma W \pm\left[\left(9|W|^{3}+2|U|^{2}\right) W+\frac{1}{9} U^{3}\right]=0
\end{aligned}
$$

where $U=3\left(3 \pi k_{1} x_{\mathrm{b}}^{2} \omega^{2}\left|\chi^{(3)}\right| / c^{2}\right)^{1 / 2} E_{1}, W=\left(3 \pi k_{1} x_{\mathrm{b}}^{2} \omega^{2}\left|\chi^{(3)}\right| / c^{2}\right)^{1 / 2} \exp (-\mathrm{i} \delta k z) E_{3}$, $\sigma=k_{3} / k_{1}, \Delta=2 k_{1} \delta k x_{\mathrm{b}}^{2}$. In these equations the absolute value of $\chi^{(3)}$ has been included into the rescaling while its sign remains as an explicit \pm in front of the Kerr nonlinearity terms. Thus Eqs. (6) with the plus sign correspond to self-focusing, while the minus sign describes the self-defocusing case.

Now we make a further rescaling $z=\beta Z$ and $x=\beta^{1 / 2} X$ (where $\beta$ is the nonlinearity-induced propagation constant shift) with a new variables $u, w$ defined as follows: $U=u \sqrt{\beta} \exp (\mathrm{i} \beta Z), W=w \sqrt{\beta} \exp (\mathrm{i} 3 \beta Z)$. Then we eventually obtain the following dimensionless equations [1]:

$$
\begin{aligned}
& \mathrm{i} u_{z}+u_{x x}-u+\left[(1 / 9)|u|^{2}+2|w|^{2}\right] u+(1 / 3) u^{* 2} w=0 \\
& \mathrm{i} \sigma w_{z}+w_{x x}-\alpha u+\left(9|w|^{2}+2|u|^{2}\right) w+(1 / 9) u^{3}=0
\end{aligned}
$$

where $u$ and $w$ are rescaled amplitudes of electric fields (fundamental frequency and its second harmonic, respectively), $\alpha$ is related to the mismatch parameter $\Delta$ by $\alpha=\sigma(3+\Delta / \beta)$, and the subscripts $z$ and $x$ denote partial derivatives with respect to the appropriate variables.

We analyze integrability of this system by means of the Painlevé test [4], then we look for conditions of solvability of the system by means of the Hirota bilinear method [5].

In Sec. 2 we show a brief outline of the Painlevé test for this case (a more detailed description was given in the communication [3]). Section 3 contains basic information on the Hirota bilinear method and the results of its application to our system (7).

\section{The Painlevé test for the 3rd harmonic equation}

In a generalization of both the property and the test to partial differential equations in $n$ independent variables [6] the solution is sought in the form of a Laurent series in powers of some expansion variable $F$ about an $(n-1)$-dimensional singularity manifold $F\left(z_{1}, z_{2}, \ldots, z_{n}\right)=0$

$$
\begin{array}{ll}
u=F^{p} \sum_{n=0}^{\infty} u_{n} F^{n}, & u^{*}=F^{q} \sum_{n=0}^{\infty} v_{n} F^{n}, \\
w=F^{r} \sum_{n=0}^{\infty} w_{n} F^{n}, & w^{*}=F^{s} \sum_{n=0}^{\infty} y_{n} F^{n},
\end{array}
$$

where separate expansions are assumed for the variables $u, w$, and their complex conjugates, $u^{*}, w^{*}$ as the conjugation relations do not hold any more when $z$ or $x$ becomes complex. 
The analysis is performed in the Kruskal gauge [7], i.e. the singularity manifold equation $F(x, z)=0$ is assumed to have the form explicitly solved for $x$, namely $x-\xi(z)=0$, while the coefficients of the Laurent series are assumed to depend on $z$ only.

The leading order exponents of all the unknowns and their complex conjugates, in (8) are

$$
p=q=r=s=-1 .
$$

Some rather unexpected complexity of the test arises from the fact that the leading order coefficients may be expressed merely in terms of solutions of a third degree equation

$$
14 R^{3}+192 R^{2}-(11520 / 7) R+4536=0,
$$

where $R=u_{0} v_{0}$.

Equation (10) has one irrational real root and two complex roots (conjugates of each other). Quoting the Cardano formulae seems to be of little use here. Taking any solution $R$ of Eq. (10) we may express the remaining leading order coefficients $v_{0}, w_{0}$, and $y_{0}$ of $u^{*}, w$ and $w^{*}$, respectively by the following equations:

$$
v_{0}=R / u_{0}, \quad w_{0}=\left(7 u_{0}^{3} v_{0}+54 u_{0}^{2}\right) /\left(252 v_{0}-63 u_{0} v_{0}^{2}\right), \quad y_{0}=v_{0}^{3} w_{0} / u_{0}^{3},
$$

where $u_{0}$ may be chosen as an arbitrary function of $z$.

A separate family of solutions to the differential equation corresponds to each of the roots of $(10)$.

The "indicial equation" which yields the indices (resonances) is an algebraic equation of the eighth degree in the index $n$. Due to symmetry of its solutions with respect to $n=3 / 2$ this complicated equation may be cast into a bit simpler form

$$
\begin{aligned}
& {\left[\left(235489234442211792 R^{2}-1549346063435380224 R\right.\right.} \\
& +3759739720852405248) s^{4}+\left(-20106193865841202280 R^{2}\right. \\
& +132284000428692645120 R-321008671765971141120) s^{2} \\
& +429167807658718777789 R^{2}-2823609296783842962144 R \\
& +6851948295917935820736](s+5 / 2)(s+3 / 2)(s-3 / 2)(s-5 / 2)=0,
\end{aligned}
$$

where $s=n-3 / 2$. Although the integer coefficients in (12) are very large, they have no common divisor except 1 .

It follows from (12) that for any choice of the initial coefficients there are four integer resonances $n \in\{-1,0,3,4\}$. The remaining four resonances are real irrational for the real initial $R=u_{0} v_{0}$ while either choice of the complex $R$ corresponds to four complex resonances (with irrational real and imaginary parts). Hence the system (7) is non-Painlevé. On the other hand, the compatibility conditions at all the integer resonances are satisfied whatever be the choice of the initial coefficients. As a result the test provides us with one arbitrary function at each of these resonances (the resonance -1 corresponds to arbitrariness of the 
expansion variable $F$ ). Thus, not being Painlevé, the system (7) still admits a four-parameter class of special solutions which are meromorphic in a vicinity of any movable singularity manifold. This holds for any choice of the parameters $\sigma$ and $\alpha$.

\section{The Hirota bilinear method}

In 1971 R. Hirota proposed an effective method of constructing multisoliton solutions of various nonlinear equations [5]. The method relies on a transformation of the original equation, by an appropriate substitution, to the form

$$
P\left(D_{x}, D_{z}\right) F \cdot G=0,
$$

where $P$ is a polynomial in the differential operators $D$ which are defined by

$$
D_{x}^{m} D_{z}^{n} F \cdot G=\left.\left(\frac{\partial}{\partial x}-\frac{\partial}{\partial x^{\prime}}\right)^{m}\left(\frac{\partial}{\partial z}-\frac{\partial}{\partial z^{\prime}}\right)^{n} F(x, z) G\left(x^{\prime}, z^{\prime}\right)\right|_{x^{\prime}=x, z^{\prime}=z}
$$

The transformation leading to (14) usually relies on expressing the unknown function as the first or second logarithmic derivative of the function $F$ inherent in (13); then $G=F$. In case of the nonlinear Schrödinger-type equations the proper transformation is

$$
u=G / F, \quad w=H / F,
$$

where $G$ and $H$ may be complex while $F$ is assumed to be real.

Such a substitution transforms our equations into

$$
\begin{aligned}
& F\left[\left(\mathrm{i} D_{z}+D_{x}^{2}-1\right) G \cdot F\right] \\
& \quad+G\left[-D_{x}^{2} F \cdot F+(1 / 9) G G^{*}+2 H H^{*}+H\left(G^{*}\right)^{2} /(3 G)\right]=0, \\
& F\left[\left(\mathrm{i} \sigma D_{z}+D_{x}{ }^{2}-\alpha\right) H \cdot F\right] \\
& \quad+H\left[-D_{x}^{2} F \cdot F+9 H H^{*}+2 G G^{*}+G^{3} /(9 H)\right]=0 .
\end{aligned}
$$

The Hirota bilinear structure of these equations may be preserved if all the four expressions in the square brackets turn to zero. However this requirement yields an overdetermined system of four equations for three unknowns $F, G, H$

$$
\begin{aligned}
& \left(\mathrm{i} D_{z}+D_{x}^{2}-1\right) G \cdot F=0, \\
& \left(\mathrm{i} \sigma D_{z}+D_{x}^{2}-\alpha\right) H \cdot F=0,
\end{aligned}
$$

and

$$
\begin{aligned}
& -D_{x}^{2} F \cdot F+(1 / 9) G G^{*}+2 H H^{*}+H\left(G^{*}\right)^{2} /(3 G)=0, \\
& -D_{x}^{2} F \cdot F+9 H H^{*}+2 G G^{*}+G^{3} /(9 H)=0 .
\end{aligned}
$$

The compatibility condition for (18) is

$$
(1 / 9) G G^{*}+2 H H^{*}+H\left(G^{*}\right)^{2} /(3 G)=9 H H^{*}+2 G G^{*}+G^{3} /(9 H),
$$

as both hand sides of this equation should be equal $-D_{x}^{2} F \cdot F$. 
After an obvious simplification, in the phase representation

$$
G=G_{0} \exp (\mathrm{i} \varphi), \quad H=H_{0} \exp (\mathrm{i} \psi)
$$

(19) turns into two equations

$$
\psi=3 \varphi \quad(\operatorname{modulo} \pi)
$$

and

$$
G_{0}=t H_{0}, \quad \text { i.e. }|u|=t|w|,
$$

where $t$ is the real solution of equation

$$
t^{3}+17 t^{2}+3 t+63=0 .
$$

Equation (23) has a unique real solution

$$
t=(1 / 3)(17+\sqrt[3]{5993-9 \sqrt{116697}}+\sqrt[3]{5993+9 \sqrt{116697}}) \approx 17.3811 .
$$

This is the only proportion between the amplitudes of the fundamental mode and its third harmonic for which the usual Hirota scheme yields soliton solutions. This result is in accordance with the condition for existence of a travelling wave solution obtained in $[1,2]$.

We should now check whether this solution is compatible with (17). Each of those equations has the typical Schrödinger conservation law for the photon number of the modes $G G^{*}$ and $H H^{*}$, respectively (obtained from the imaginary part of the equation)

$$
\begin{aligned}
& \frac{\partial}{\partial z} \frac{G G^{*}}{F^{2}}+2 \frac{\partial}{\partial x}\left(\frac{G G^{*}}{F^{2}} \frac{\partial}{\partial x} \varphi\right)=0, \\
& \sigma \frac{\partial}{\partial z} \frac{H H^{*}}{F^{2}}+2 \frac{\partial}{\partial x}\left(\frac{H H^{*}}{F^{2}} \frac{\partial}{\partial x} \psi\right)=0 .
\end{aligned}
$$

When $G, H$, and their complex conjugates are proportional (22) while the phases $\varphi, \psi$ satisfy (21) then the above equations are compatible iff

$$
\left(\frac{\sigma}{3}-1\right) \frac{\partial}{\partial z} \frac{G G^{*}}{F^{2}}=0
$$

which admits nontrivial solutions only if

$$
\sigma=3
$$

Examination of the real parts of (17) when all these conditions $(21,22,27)$ are satisfied yields an equation for the phase $\varphi$

$$
\varphi_{z}(x, z)-2\left[\varphi_{x}(x, z)\right]^{2}=(1-\alpha) / 8 .
$$

Within its range of uniqueness the initial value problem in $z$ for (28) may be readily solved by the method of characteristics. Let

$$
p=\varphi_{x}\left(x_{0}, 0\right), \quad q=(1-\alpha) / 8+2\left[\varphi_{x}\left(x_{0}, 0\right)\right]^{2},
$$


where $x_{0}$ is the solution of

$$
x=x_{0}-4 \varphi_{x}\left(x_{0}, 0\right) t .
$$

Then, within such an interval of $z$, for which the solution of (30) is unique, Eq. (28) is satisfied by

$$
\varphi(x, z)=\varphi\left(x_{0}, 0\right)-\left(x-x_{0}\right)^{2} /(8 z)+(\alpha-1) z / 8 .
$$

The apparent singularity of (31) at $z=0$ is removable for initial conditions in $\mathcal{C}^{2}$, namely it follows from (30) that $x-x_{0} \rightarrow 0$ when $z \rightarrow 0$.

It follows from the equation of characteristics (30) that the characteristics of the phase equation (28) are straight lines; $\varphi_{x}$ is constant along these characteristics. On the other hand $\varphi_{x}$ is connected with squares of the amplitudes $G G^{*} / F^{2}$ and $H H^{*} / F^{2}$ by Eq. (25). As a result the amplitudes also do not vary along the same rectilinear characteristics. This is the typical property of single solitary waves rather than multisoliton solutions.

\section{Conclusion}

We have examined the third harmonic equations using the Painlevé test and got some preliminary results from the Hirota bilinear formalism. The Painlevé test has shown that in general the system does not have the Painlevé property (like the second harmonic equation) but (unlike the second harmonic equation) it has a 4-parameter class of meromorphic solutions without any extra constraints on its parameters. The Hirota bilinear formalism yields two conditions for the fundamental mode and its third harmonic: one for their phases and another one for their amplitudes. They allow for nontrivial solutions if $\sigma=3$. For this value of $\sigma$ we have obtained the explicit solution for the phase by the method of characteristics. These characteristics, which also prove to be the lines of constant amplitude, are straight lines. As such evolution does not leave room for soliton interaction, we conclude that the only possible soliton solutions are single envelope solitons of the nonlinear Schrödinger type with the phase increasing linearly both in $x$ and $z$. However these solutions are very special. A thorough analysis of the system by means of generalizations of the Hirota method [8] may help to go beyond these limitations.

\section{References}

[1] R.A. Sammut, A.V. Buryak, Y.S. Kivshar, J. Opt. Soc. Am. B 15, 1488 (1998).

[2] Yijiang Chen, Phys. Rev. A 50, 5145 (1994).

[3] V. Cao Long, P.P. Goldstein, M. Trippenbach, D. Nguyen Tien, in: Proc. Nation. Conf. on Theoretical Physics, Sam Son (Vietnam), August 2003, in press.

[4] R. Conte, in: The Painlevé Property One Century Later, Ed. R. Conte, Springer Verlag, New York 1999, ch. 3, p. 77; also M. Musette, ibidem, ch. 8, p. 517 . 
[5] R. Hirota, Phys. Rev. Lett. 27, 1192 (1971); R. Hirota, in: Solitons, Eds. R.K. Bullough, P.J. Caudrey, Springer Verlag, Berlin 1980, p. 157.

[6] J. Weiss, M. Tabor, G. Carnevale, J. Math. Phys. 24, 522 (1983); J. Weiss, J. Math. Phys. 25, 2226 (1984).

[7] M. Jimbo, M.D. Kruskal, T. Miwa, Phys. Lett. A 92, 59 (1982).

[8] J. Hietarinta, Int. J. Mod. Phys. A 12, 43 (1997); B. Grammaticos, A. Ramani, J. Hietarinta, Phys. Lett. A 190, 65 (1994). 